\title{
Enhanced second harmonic generation from InAs nano-wing structures on silicon $\dagger$
}

Cite this: Nanoscale, 2013, 5, 10163

Received 14th June 2013

Accepted 23rd August 2013

\author{
Emanuele Francesco Pecora, ${ }^{a}$ Gary F. Walsh, ${ }^{\text {ab }}$ Carlo Forestiere, ${ }^{\text {ac }}$ Alex Handin, ${ }^{a}$ \\ Eleonora Russo-Averchi, ${ }^{d}$ Anna Dalmau-Mallorqui, ${ }^{d}$ Ignasi Canales-Mundet, ${ }^{b}$ \\ Anna Fontcuberta i Morral ${ }^{d}$ and Luca Dal Negro*a
}

DOI: $10.1039 /$ c3nr03083k

www.rsc.org/nanoscale

We demonstrate morphology-dependent second-harmonic generation (SHG) from InAs V-shaped nanomembranes. We show SHG correlation with the nano-wing shape and size, experimentally quantify the SHG efficiency, and demonstrate a maximum SHG enhancement of about 500 compared to the bulk. Experimental data are supported by rigorous calculations of local electromagnetic field spectra.

\section{Introduction}

Nonlinear optical phenomena have been recently exploited in order to demonstrate photon manipulation on a single chip, where new functionalities such as parametric down-conversion, optical broadband routing, and multiple frequency generation can potentially be integrated..$^{1-4}$ In particular, the second harmonic generation (SHG) process, which originates from the second-order optical susceptibility $\chi^{(2)}$ of materials, enables the conversion of two photons (usually in the near-IR wavelength range) into a single photon of doubled frequency. Many different semiconductor materials exhibit large secondorder susceptibility, but they cannot easily be integrated with the widespread Si-based microelectronics technology. On the other hand, bulk Si possesses only a negligible $\chi^{(2)}$ due to its centrosymmetric crystal structure, which makes it unsuitable for efficient nonlinear optical generation on a chip. Nevertheless, a significant amount of research activities have

${ }^{a}$ Department of Electrical and Computer Engineering \& Photonics Center, Boston University, 8 Saint Mary Street, Boston, Massachusetts 02215, USA. E-mail: dalnegro@bu.edu

${ }^{b}$ Nanomaterials Science Team, U.S. Army NSRDEC, Kansas Street, Natick, Massachusetts 01760, USA

${ }^{c}$ Department of Electrical Engineering and Information Technology, Università degli Studi di Napoli Federico II, via Claudio 21, Napoli 80125, Italy

${ }^{d}$ Laboratoire des Matériaux Semiconducteurs, Ecole Polytechnique Fédérale de Lausanne, 1015 Lausanne, Switzerland

$\uparrow$ Electronic supplementary information (ESI) available: See DOI: $10.1039 / \mathrm{c} 3 \mathrm{nr} 03083 \mathrm{k}$ demonstrated promising strategies to boost optical nonlinearities in Si-based materials systems. Recently, enhanced secondorder nonlinearity in Si has been demonstrated by a number of approaches including higher-order multipolar processes, ${ }^{5}$ the breaking of crystal symmetry at interfaces, the engineering of strain fields, ${ }^{6-12}$ or the development of substoichiometric silicon nitride thin films. ${ }^{13}$ An alternative strategy relies on the integration atop $\mathrm{Si}$ technology of non-centrosymmetric semiconductor nanostructures that feature very efficient optical nonlinear coefficients, thus potentially enabling a number of novel device applications. In particular, III-V semiconductors monolithically grown on Si-compatible substrates are already utilized for specific optoelectronics applications due to their direct band-gaps and high carrier mobilities. ${ }^{14-16}$ Moreover, III-V semiconductor materials can be synthesized in a large variety of nanostructures with different sizes, shapes, architectures, and compositions, resulting in largely tunable quantum size effects and morphology-driven optical resonances that permit to flexibly engineer light-matter interactions. In particular, three-dimensional structures such as nanowires, ${ }^{17-21}$ nanotrees, ${ }^{22}$ nanorings, ${ }^{23}$ nanoflowers, ${ }^{24,25}$ or nanomembranes ${ }^{26}$ have shown significant advantages over traditional quantum dots and quantum wells due to their ability to support tunable resonances that enhance their optical properties. ${ }^{27-30}$ A number of studies have been performed on the linear optical properties of III-V nanostructures, but little is known on the interplay between morphologydependent structural resonances and their nonlinear optical properties.

In this paper, we systematically investigate the second harmonic generation in InAs V-shaped nanomembranes, dubbed nano-wings, grown epitaxially on (001) Si substrates. By investigating a number of samples fabricated with varying geometrical parameters, we correlate the measured SHG signal and efficiency to the shape and size of the nano-wings, demonstrating a maximum SHG signal enhanced by about 500 times compared to a reference InAs bulk sample due to the excitation of structural resonances with large local field enhancement at the pump wavelength. 


\section{Methods}

\section{Growth}

The InAs membranes have been grown by molecular beam epitaxy in a DCA P600 system. We have used (001) p-doped silicon wafers with a resistivity of $0.1-0.5 \Omega \mathrm{cm}$ on which we deposited a $20 \mathrm{~nm}$ thick $\mathrm{SiO}_{2}$ layer of thermal oxide as a growth mask. Electron beam lithography has been used to pattern holes with diameters ranging between 100 and $180 \mathrm{~nm}$ and inter-hole distances ranging from 200 to $2000 \mathrm{~nm}$ in a ZEP resist. The pattern has been then transferred on the oxide layer by a $12 \mathrm{~s} 7: 1$ buffered HF wet etch. Prior to the introduction to the MBE reactor, the wafer underwent a further $2 \mathrm{~s}$ dip in the buffered HF solution in order to guarantee a perfectly clean and oxide-free surface in the holes. The substrates were subsequently degassed at $600{ }^{\circ} \mathrm{C}$ for $2 \mathrm{~h}$ in $\mathrm{UHV}$ and then again at $770{ }^{\circ} \mathrm{C}$ for $30 \mathrm{~min}$ when transferred to the growth chamber to further remove any possible surface contaminants. The growth was carried out at a nominal In growth rate of $0.2 \AA \mathrm{s}^{-1}$, at a temperature of $520^{\circ} \mathrm{C}, \mathrm{As}_{4}$ partial pressure of 0.8 and $1.15 \times 10^{-5}$ Torr (growth time respectively of $1 \mathrm{~h}$ and $2 \mathrm{~h}$ ) and with $7 \mathrm{rpm}$ rotation.

\section{Optical set-up}

Second harmonic generation has been studied using a modelocked high power ultrafast Ti:sapphire laser (MaiTai HP SpectraPhysics, 150 fs pulse width) coupled with an electrooptic pulse picker to set the repetition rate at $20 \mathrm{MHz}$. The average pumping power was $8 \mathrm{~mW}$, laser light was horizontal polarized, and the excitation wavelength in the range 700-900 nm. We ensured that no thermal oxidation occurred under these conditions, and measures were repeatable in the same spot after sweeping the pump power several times. The pump beam was focused on the sample using a $20 \times$ microscope objective, unless otherwise specified in the text. The same objective was used to collect the back-reflected light. A long-pass filter was placed before the sample to avoid any unrelated signal at the second harmonic wavelengths. The sample output was collected through a standard telescoping lens arrangement and coupled into an f/4 monochromator (Cornerstone 260). The pump light was rejected by a short-pass filter placed in front of the monochromator slits. The SHG spectra have been detected with a lock-in amplifier (Oriel Merlin) coupled to a low-light photomultiplier tube (PMT, Oriel Instrumentation 77348). The SHG output power has been estimated considering the PMT responsivity, the measured transmittivity of all the optical components and the light coupling efficiency into the monochromator, and also directly measured through a highly sensitive power meter detector (Newport 918D-UVOD3), as reported elsewhere. ${ }^{13}$ The same set-up was also used for imaging and aligning the sample in order to ensure overlap between the laser beam-illuminated area and the nanostructure area in the sample. For this purpose, the output of the objective was sent also to a visible-sensitive camera and the alignment was verified before every measure. A schematic of the experimental set-up is sketched in the ESI, Fig. SI1. $\dagger$

\section{Modeling}

Electromagnetic scattering calculations were performed using a Surface Integral Formulation of Maxwell's equations. They very effectively treat the scattering from arbitrary shaped particles since they require only the discretization of the spatial domain occupied by the scatterers, while the radiation conditions are automatically satisfied. A detailed comparison of the accuracy of several Surface Integral Formulations in both the far and near field zone is reported elsewhere. ${ }^{31}$ The calculations performed in this paper are based on the PMCHWT (Poggio, Miller, Chang, Harrington, and $\mathrm{Wu}$ ) formulation. ${ }^{32,33}$ In this work, meshes with about $4 \mathrm{k}$ edges have been used to numerically solve the scattering problem.

\section{Results and discussion}

\section{Nanomembranes growth and structural characterization}

Fig. 1 shows $20^{\circ}$ tilted scanning electron microscopy (SEM) images of typical InAs membranes grown at $520{ }^{\circ} \mathrm{C}$, nominal In growth rate of $0.2 \AA \mathrm{S}^{-1}$ and under $\mathrm{As}_{4}$ partial pressure of $0.8 \times$ $10^{-5}$ Torr (a $-1 \mathrm{~h}$ growth) and $1.15 \times 10^{-5}$ Torr $(\mathrm{b}-2 \mathrm{~h}$ growth). The images reveal the peculiar shape of the nanostructures with two preferred directions of elongation. The structural properties of these nanosctructures have already been discussed elsewhere. ${ }^{26,34}$

They present a mixture of wurtzite and zinc-blende structure, and extend along two opposite $<111>$ B directions, forming flat $\{110\}$ walls. In particular, in a top-down projection, we found two comparable distribution of membranes, extending respectively along the $\langle 1-10\rangle$ and $\langle 110\rangle$ directions. The direction perpendicular to the substrate and parallel to the incident light is the (100). As the excitation beam is linearly polarized, we will always observe a combination of the longitudinal and transverse mode on the nanostructures. As described in the experimental section, the membranes are positioned in regular holes arrays in $\mathrm{a} \mathrm{SiO}_{2}$ mask with diameter changing from 100 to $180 \mathrm{~nm}$ and inter-hole distance (pitch) changing from 200 to $2000 \mathrm{~nm}$. Fig. 1(c) and (d) show the effect of the pitch and the opening size on the size of membranes grown under the conditions mentioned above (in blue: $\mathrm{As}_{4}$ partial pressure of 0.8 $\times 10^{-5}$ Torr for $1 \mathrm{~h}$, in red: $\mathrm{As}_{4}$ partial pressure of $1.15 \times 10^{-5}$ Torr for $2 \mathrm{~h}$ ). Both the samples are characterized by a significant length variation as a function of the pitch (Fig. 1(c)). This dependence is characterized by two separate growth regimes as it has been found by other groups for nanowires grown on pattern: ${ }^{35-37}$ for pitches below $800 \mathrm{~nm}$ the length increases with the pitch, while above that threshold the length saturates. The switch between the two regimes is determined by the indium surface diffusion length on the $\mathrm{SiO}_{2}$. A detailed description of this mechanism is discussed in a separate work. ${ }^{34}$ The effect of the hole size on the morphology of the membranes is described in Fig. 1(d): the width of the membranes increases by increasing the opening sizes. As noted in Conesa-Boj et al. ${ }^{26}$ the membranes originate from the two opposite facets of a rectangular pyramidal island nucleus, the size of which is in turn determined by the opening size. As for the dependence of the 

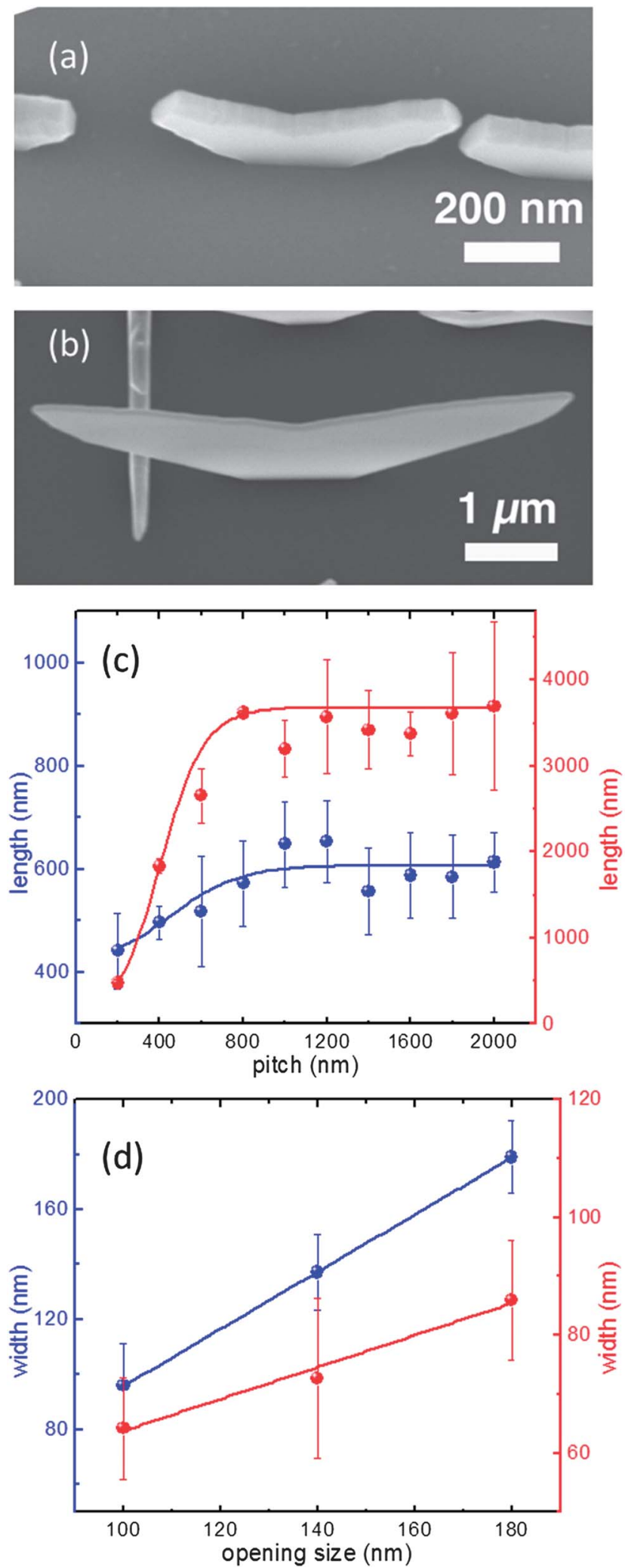

Fig. 1 Scanning electron microscopy (SEM) tilted $\left(20^{\circ}\right)$ images of typical InAs membranes grown at $520^{\circ} \mathrm{C}$, nominal In growth rate of $0.2 \AA \mathrm{s}^{-1}$ and under $\mathrm{As}_{4}$ partial pressure of $0.8 \times 10^{-5}$ Torr ( $\mathrm{a}-1 \mathrm{~h}$ growth) and $1.15 \times 10^{-5} \mathrm{Torr}(\mathrm{b}-2 \mathrm{~h}$ growth). Effect of the pitch on the length of InAs membranes: the length increases by increasing the pitch and saturates (c). Plot of the membranes width as a function of the opening size: the width increases by increasing the opening size (d). length on the pitch, a detailed description of this behavior will be provided in a dedicated study.

\section{Electromagnetic modeling}

Using the accurate surface integral equation (SIE) method, ${ }^{31-33}$ we investigate the scattering and near field behaviour of nanowings as a function of incident wavelength, first by varying the length of the nano-wing for fixed widths and then by varying the width for fixed lengths. Engineering the distinctive scattering and near-field response of dielectric nano-wing structures is of importance for tailoring their linear and nonlinear optical properties, as it will be demonstrated in the next section. We excited the nano-wing with a plane wave of unit intensity propagating in the negative $z$-direction for two different polarizations, longitudinal ( $x$-polarized with reference to Fig. 2) and transverse ( $y$-polarized) to the length of the nano-wing. Fig. 2(a) and (b) show the scattering efficiency as a function of wavelength for different nano-wing lengths with a fixed width of $75 \mathrm{~nm}$ for longitudinal and transverse excitation respectively. For both longitudinal and transverse excitation, we can see a resonant behaviour controlled by the geometrical parameters of the nano-wing. As the length increases from $1850 \mathrm{~nm}$ to 3615 $\mathrm{nm}$, the peak value of the scattering efficiency also increases, and in the case of longitudinal polarization undergoes a substantial red-shift. Fig. 2(c) and (d) show the scattering efficiency as a function of wavelength for different nano-wing width with a fixed length of $1600 \mathrm{~nm}$ for longitudinal and transverse excitation respectively. For longitudinal polarization, as the width increases from $50 \mathrm{~nm}$ to $160 \mathrm{~nm}$ the peak value decreases, whereas we found no significant trend for the transverse polarization. For both polarizations we observe a strong redshift. In conclusion, we have shown a resonant scattering behaviour of the nano-wing driven by its geometrical parameters. We now investigate the near-field distribution of the nanowing resonances, and show that they can significantly enhance the local intensity of incident electric fields. Fig. 3(a) and (b) show the total electric field (sum of incident and scattered field) plotted on the surface of the nano-wing for the longitudinal and transverse modes respectively. We calculated the surface field with a single incident wavelength of $800 \mathrm{~nm}$ for a nano-wing with a length of $1150 \mathrm{~nm}$ and a width of $75 \mathrm{~nm}$. When the incident field is longitudinally polarized, the total field on the surface of the nano-wing is strongly localized at the two tips and along the edges of the faces. In the case of a transverse incident polarization the total field is more uniformly distributed on the vertical faces of the nano-wing, although it assumes lower values compared to the longitudinal excitation configuration. Fig. 3(c) and (d) show the average of the surface electric field as a function of wavelength for different nanowing lengths with a fixed width of $75 \mathrm{~nm}$ for longitudinal and transverse excitation respectively. For longitudinal excitation, at a wavelength of $800 \mathrm{~nm}$, as the length increases from 1850 $\mathrm{nm}$ to $3615 \mathrm{~nm}$, the average electric field decreases. Our analysis unveils that a clear trend exists: the shortest nanowing features the largest average surface electric field. For transverse excitation, no clear trend exists for the investigated 
longitudinal mode

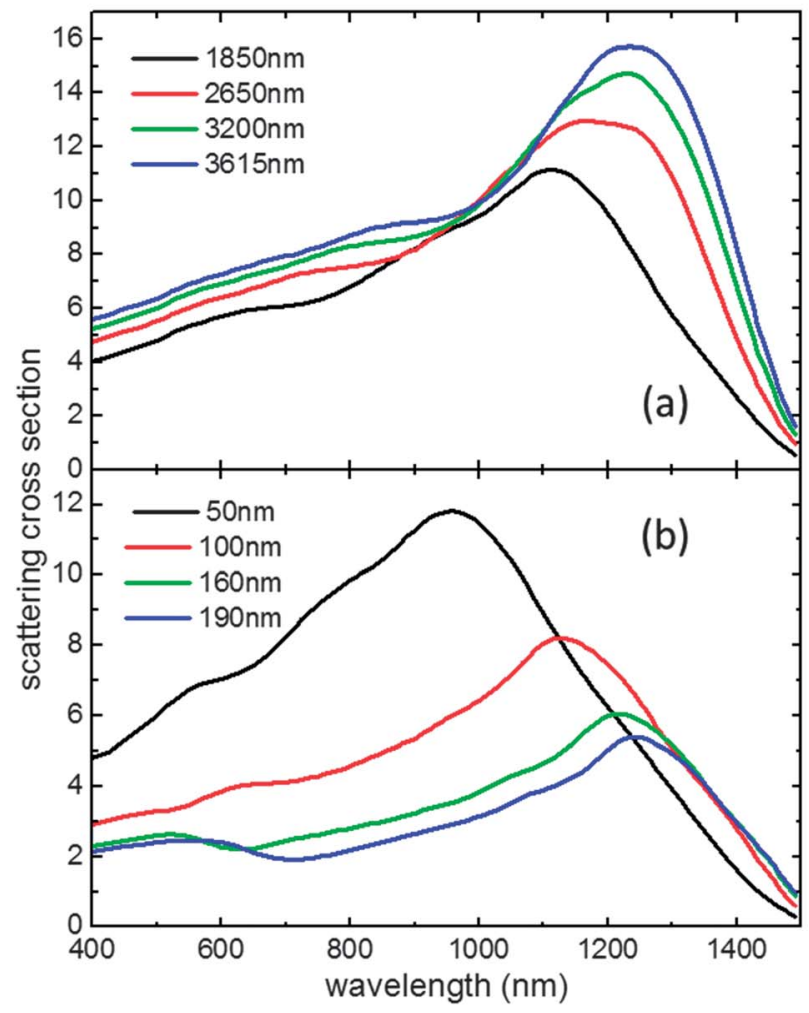

transverse mode

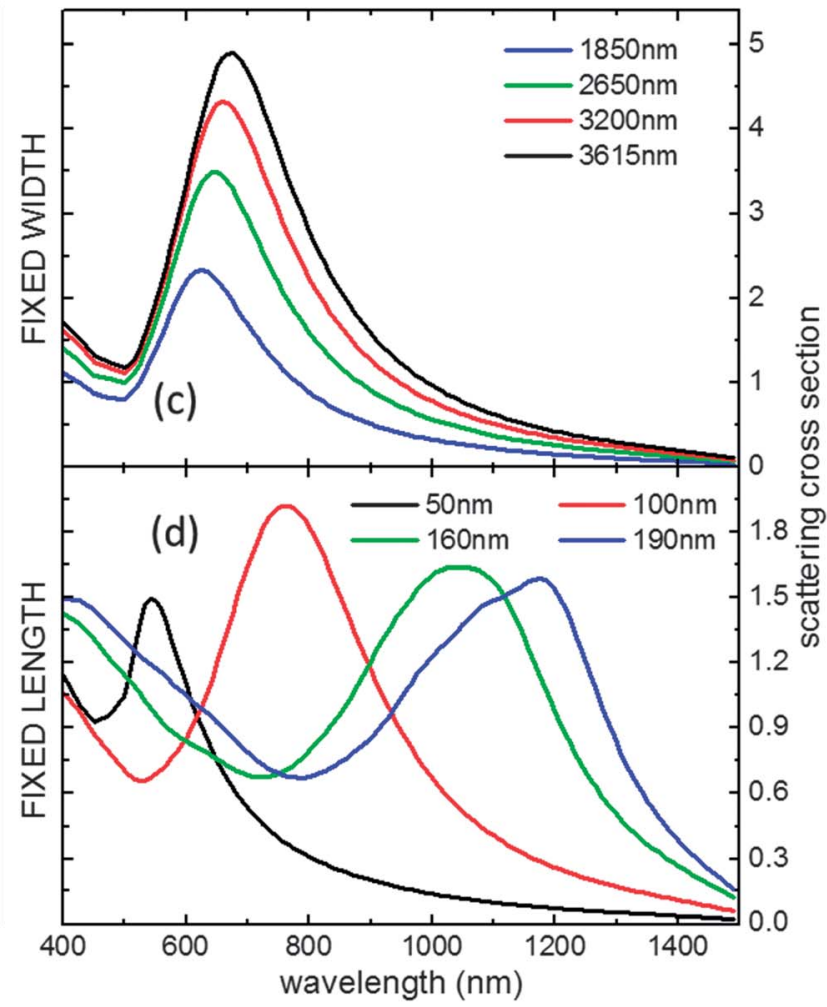

Fig. 2 Spectrum of the scattering efficiency as a function of wavelength for different nano-wing lengths with a fixed width of $75 \mathrm{~nm}$ for longitudinal (a) and transverse excitation (b) respectively. Spectrum of the scattering efficiency as a function of wavelength for different nano-wing widths with a fixed length of $1600 \mathrm{~nm}$ for longitudinal (c) and transverse (d) excitation. The nano-wing is excited with a plane wave of unit intensity propagating in the negative $z$-direction.

set of parameters. The structure with largest average electric field has a length of $800 \mathrm{~nm}$ while the other structures have approximately the same field strength.

\section{Second harmonic experiments}

In this section we investigate the second harmonic generation (SHG) from nano-wing samples supporting tunable near-field and scattering resonances spectrally overlapping the pump wavelength. The possibility to significantly enhance and control the SHG of nano-wings by tailoring their nanoscale morphology will be experimentally demonstrated. The second harmonic generation takes place in both the surface and the bulk of the InAs nanostructures. Both bulk and surface sources of nonlinearity can be strongly influenced by the local distribution of the electric field on the surface and within the bulk of the InAs nanostructures, as a result of their resonant optical response. We notice that, in the case of crystalline InAs, materials, theoretical considerations ${ }^{44}$ suggest that the bulk contribution to the second harmonic generation dominates over the surface contribution. This has been confirmed by experimental results of SHG in planar InAs slabs..$^{38}$ On the other hand, little is known on the relative contributions of bulk versus surface nonlinear contributions in resonant nanostructures with non-spherical shapes, such as the investigated nano-wings, and a systematic experimental study is proposed in this section. In Fig. 4(a) we show representative second harmonic (SH) spectra recorded from a sample with a $500 \mathrm{~nm}$ span of the nano-wings (from here on referred to as the length of the nano-wing), and wing thicknesses in the range between $95 \mathrm{~nm}$ and $180 \mathrm{~nm}$. It is worth noting that the average pump power used to generate the $\mathrm{SH}$ signal in the paper was only $8 \mathrm{~mW}(800 \mathrm{~nm}$ excitation wavelength). The SH signal detected from a bulk InAs wafer under exactly the same experimental conditions is also reported as a reference (spectrum has been multiplied by 20 to be visible in the plot). In particular, our substrate is (001) oriented and we measured the azimuthal angle of the $\mathrm{SH}$ emission in order to identify the angle corresponding to the highest SH intesity. ${ }^{38} \mathrm{We}$ show in Fig. SI $2 \dagger$ the trend with the azimuthal angle of our data. The measured signal from the nanostructures is consistently at least one order of magnitude higher than from the bulk material. Moreover, it is important to observe that no scaling factor has been applied to these data, despite the illuminated material volume is much smaller in the nano-wing samples compared to the reference bulk material. This demonstrates that the V-shape morphology of the nano-wings plays a fundamental role in enhancing the SH emission. Furthermore, we observe a clear trend between the SH intensity and the thickness of the nanowings, establishing a correlation between the structural parameters of the wings and their enhanced nonlinear optical responses. We discovered that the strongest $\mathrm{SH}$ signal is generated by the thinnest nano-wings. In addition, in Fig. 4 we 


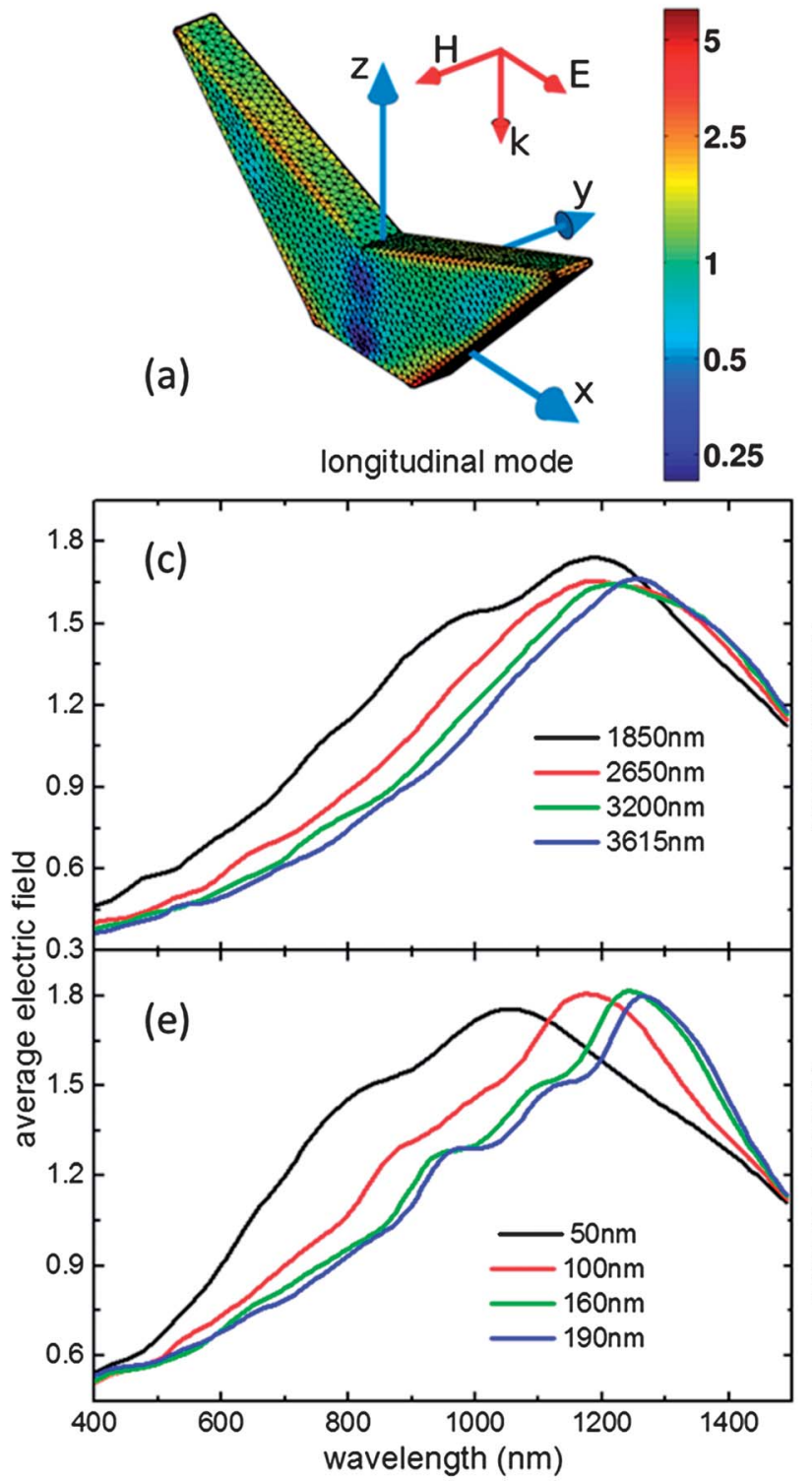

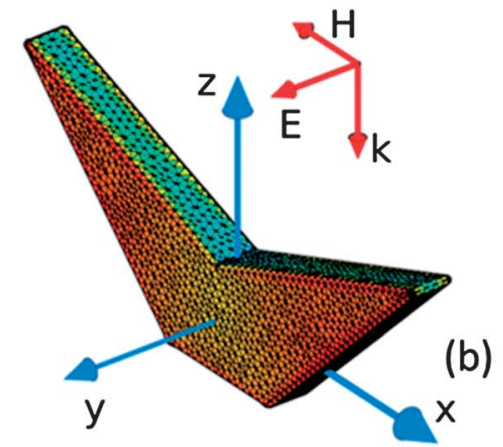

(b)

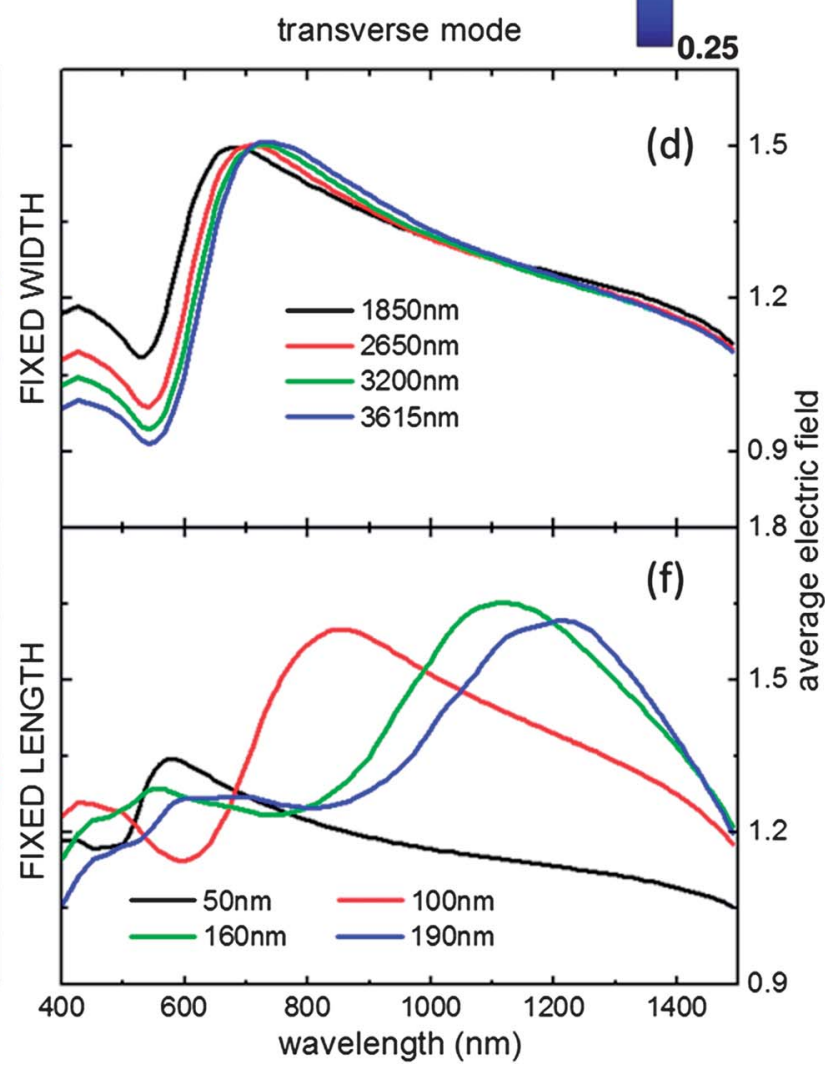

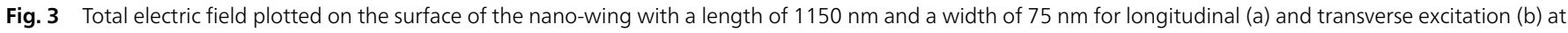

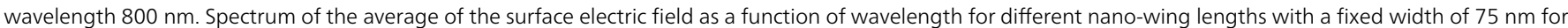

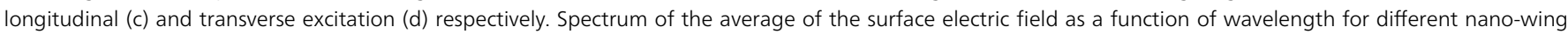

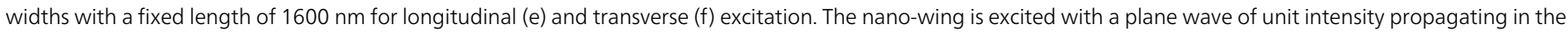
negative $z$-direction.

demonstrate the large tunability of the nonlinear optical properties of nano-wings. Specifically, in Fig. 4(b) we show the second harmonic spectra obtained under different excitation wavelengths spanning a broad range (740-1040 nm). Data are normalized for the photon flux at each wavelength and for our collection system response in order to be comparable. ${ }^{39}$ Consistent with the nonlinear nature of the SHG process, the $\mathrm{SH}$ spectra are always peaked at twice the frequency of the pumping photons. Moreover, we observe a monotonically increase of the SH signal as the wavelength increases. In order to fully demonstrate the nonlinear nature of this process, we show in Fig. 4(c) the pump power dependence of the measured $\mathrm{SH}$ intensity detected at $400 \mathrm{~nm}$. Similar trends have been measured for all the samples. The double logarithmic plot of the pump-power dependence is fitted by a continuous line (Fig. 4(c)) of almost quadratic slope $(2.1 \pm 0.1)$, clearly proving the second-order nonlinear nature of the $\mathrm{SH}$ emission process.

The role of the shape and the geometrical parameters of the InAs nano-wings is elucidated in Fig. 5. We measured the $\mathrm{SH}$ emission and also calculated the local electric field enhancement (under plane-wave excitation) at the pump frequency for a broad range of thicknesses and lengths of the nano-wings, and found a clear correlation between their respective trends. Fig. 5(a) shows the measured SHG intensity as a function of the nano-wing width, for different values of the nano-wing lengths. Data are normalized at the maximum value for a better 

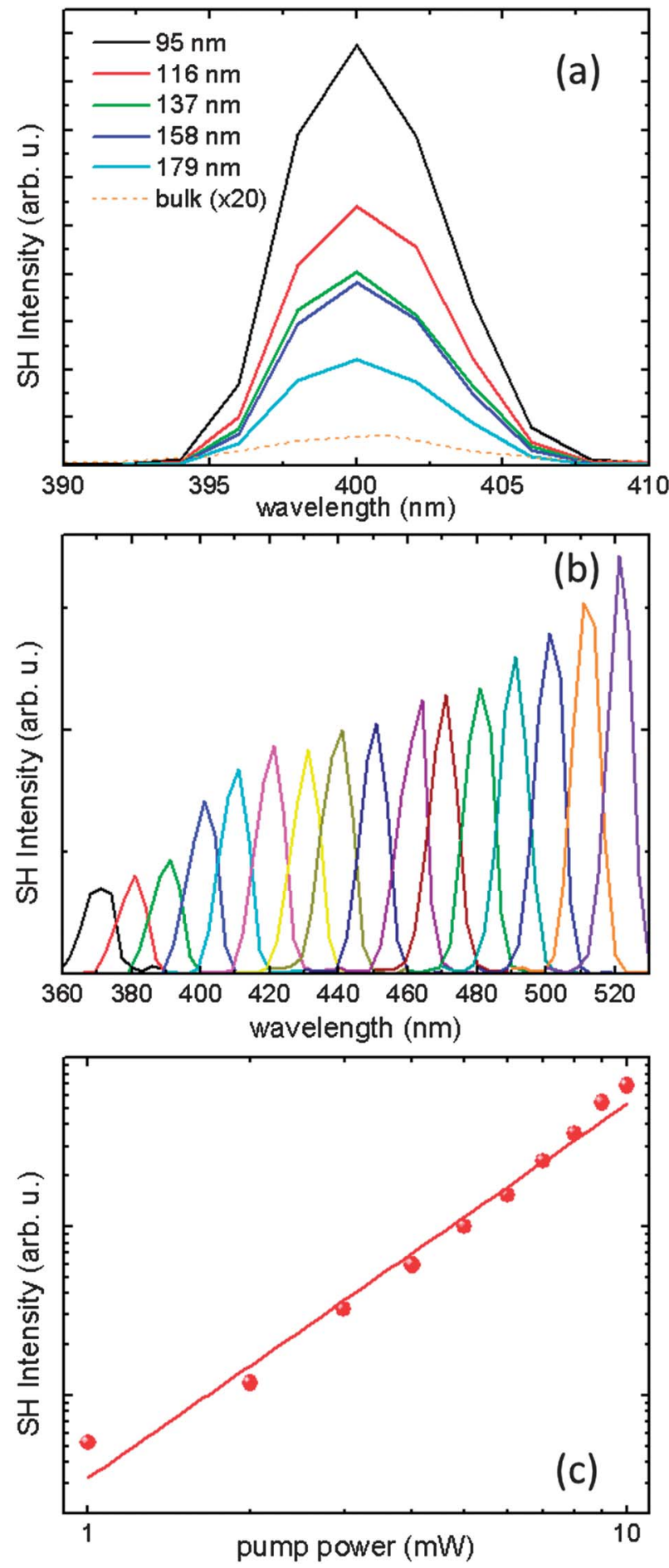

Fig. 4 (a) Second harmonic spectra from a sample with $500 \mathrm{~nm}$-long nanowings, and thicknesses in the range between $95 \mathrm{~nm}$ and $180 \mathrm{~nm}$. Average pumping power was $8 \mathrm{~mW}$, excitation wavelength $800 \mathrm{~nm}$. The SH spectrum (multiplied by 20) from a bulk InAs wafer is also reported as a reference. (b) $\mathrm{SH}$ excitation spectra from a sample with $500 \mathrm{~nm}$-long and $137 \mathrm{~nm}$-thin nano-wings. Average pumping power was fixed at $8 \mathrm{~mW}$. (c) Pump power dependence of the $\mathrm{SH}$ intensity detected at $400 \mathrm{~nm}$ (log-log scale). Continuous line is the linear fit of the data, exhibiting a slope of $2.1 \pm 0.1$.

comparison. Continuous lines are guides to the eyes. We can observe that the SHG intensity is strongly dependent on the nano-wing width, and it decreases for the thickest structures.
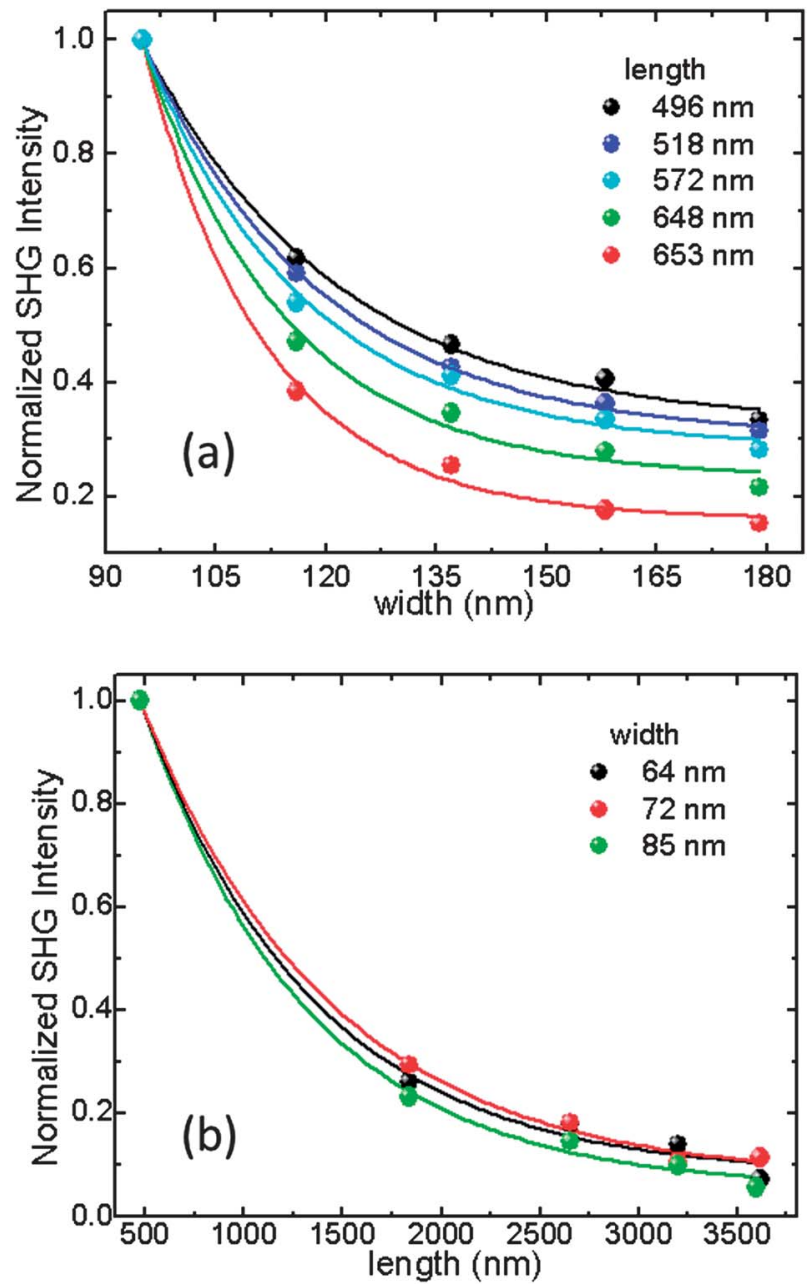

Fig. 5 SHG intensity as a function of the membrane width, for different fixed nano-wing lengths (a), and as a function of length for fixed widths (b). Data are normalized at the maximum value for a better comparison. Continuous lines are guide to eyes.

Trends are consistent for all the length values, although the data scales at different rates that depend on the length of the wings. In Fig. 5(b) we show the normalized SHG intensity as a function of wing length for fixed values of the widths. We observe a decrease of the SH intensity as the wing length increases.

Additionally, we measured for each sample the SHG output power to quantify the SH generation efficiency (pumping power has been kept at $8 \mathrm{~mW}$ for all experiments). We report the $\mathrm{SH}$ efficiency for samples with different width, length fixed at 572 $\mathrm{nm}$ (Table 1), and with different length, width fixed at $72 \mathrm{~nm}$ (Table 2). Data have to be compared with efficiencies reported in literature from other nanostructures. ${ }^{12,13,40-43}$ The measured efficiency for the bulk InAs bulk substrate is reported as a reference. The SH enhancement due to the nano-wing shaped samples is measured and it features a maximum enhancement of the SH generation of approximately a factor 500 for the thinnest $(72 \mathrm{~nm})$ and shortest $(475 \mathrm{~nm})$ nanostructures. We attribute this SHG enhancement to the resonantly enhanced electric field intensity at the pump wavelength, associated to the excitation of the distinctive structural resonances of nano-wings. 
Table 1 Measured SH efficiency and SH enhancement compared to bulk InAs for nano-wings with different width and fixed length (572 nm)

\begin{tabular}{lrr}
\hline Width $(\mathrm{nm})$ & SHG efficiency & SHG enhancement \\
\hline 95 & $1.821 \times 10^{-4}$ & 303 \\
116 & $1.129 \times 10^{-4}$ & 188 \\
137 & $8.50 \times 10^{-5}$ & 141 \\
158 & $7.88 \times 10^{-5}$ & 131 \\
179 & $4.60 \times 10^{-5}$ & 76 \\
Bulk & $6 \times 10^{-7}$ & 1 \\
\hline
\end{tabular}

Table 2 Measured SH efficiency and SH enhancement compared to bulk InAs for nano-wings with different length and fixed width $(72 \mathrm{~nm})$

\begin{tabular}{lrc}
\hline Length $(\mathrm{nm})$ & SHG efficiency & SHG enhancement \\
\hline 475 & $2.932 \times 10^{-4}$ & 488 \\
1837 & $5.49 \times 10^{-5}$ & 91 \\
2655 & $5.33 \times 10^{-5}$ & 88 \\
3200 & $3.13 \times 10^{-5}$ & 52 \\
3615 & $1.15 \times 10^{-5}$ & 19 \\
Bulk & $6 \times 10^{-7}$ & 1 \\
\hline
\end{tabular}

On the other hand, based on the calculated scattering cross sections of nano-wings (see Fig. 2), the role of scattering resonances at the SHG wavelength is negligible with respect to the pump wavelength. While a rigorous theoretical analysis of the nonlinear generation in nano-wing structures is beyond the scope of this paper, we believe that our experimental demonstration of resonant control and SHG enhancement in InAs nano-wing structures can result in novel functionalities uniquely enabled by the engineering of resonant electromagnetic fields at the nanoscale.

\section{Conclusions}

In this work we demonstrated tunable and strongly enhanced (up to 500 times) second harmonic generation from InAs nanowings of varying geometrical parameters monolithically grown on a Si substrate. We studied the resonant behaviour of nanowing structures by the rigorous calculation of morphology dependent scattering resonances and near-field electromagnetic spectra, obtained using the surface integral equation technique. We experimentally investigated the second harmonic generation in this novel type of resonant nanostructures and experimentally quantified their SHG efficiency, and show a direct correlation with the shape and size of the nano-wings. The monolithic integration of III-V semiconductor nanostructures with enhanced nonlinear optical properties on a Si platform paves the way to novel device structures leveraging with tunable optical resonances for nonlinear photon generation on a chip.

\section{Acknowledgements}

This work was supported by the AFOSR program "Emitters for High Density Information Processing using Photonic-
Plasmonic Coupling in Coaxial Nanopillars" under Award no. FA9550-13-1-0011, and by the U.S. Army Research Laboratory through the Collaborative Research Alliance (CRA) for MultiScale multidisciplinary Modeling of Electronic materials (MSME). G.W. acknowledges the U.S. Army Natick Soldier Research, Development, and Engineering Center, and the SMART Scholarship Program. NSRDEC PAO\# U13-254. ERC Starting Grant UpCon, NCCR-QSIT, SNF through grant 143908. The authors thank P. Caroff and X. Wallart for sharing the InAs reference sample. E.F.P. and G.W. performed the experiments and analyzed data. A.H. and C.F. carried out the simulations. E.R.A. and A.D.M. fabricated the samples; I.C.M. and E.R.A. characterized their dimensions. E.F.P. and L.D.N. wrote the manuscript in collaboration with all the authors. L.D.N. conceived the scientific question; L.D.N. and A.F.M. planned the set of experiments. All authors have given approval to the final version of the manuscript.

\section{Notes and references}

1 R. L. Sutherland, Handbook of Nonlinear Optics, CRC Press, 2003.

2 J. Leuthold, C. Koos and W. Freude, Nat. Photonics, 2010, 4, 535.

3 M. A. Foster, A. C. Turner, J. E. Sharping, B. S. Schmidt, M. Lipson and A. L. Gaeta, Nature, 2006, 441, 960.

4 A. M. Armani, R. P. Kulkarni, S. E. Fraser, R. C. Flagan and K. J. Vahala, Science, 2007, 317, 783.

5 D. Guidotti and T. Driscoll, Nuovo Cimento Soc. Ital. Fis., D, 1986, 8, 385.

6 S. V. Govorkov, V. I. Emel'yanov, N. I. Koroteev, G. I. Petrov, I. L. Shumay, V. V. Yakovlev and R. V. Khokhlov, J. Opt. Soc. Am. B, 1989, 6, 1117.

7 J. Y. Huang, Jpn. J. Appl. Phys., 1994, 33, 3878.

8 M. Falasconi, L. C. Andreani, A. M. Malvezzi, M. Patrini, V. Mulloni and L. Pavesi, Surf. Sci., 2001, 481, 105.

9 J. Zhao, Q. Chen, Z. Chen, G. Jia, W. Su, Y. Jiang, Z. Yan, T. V. Dolgova, O. A. Aktsipetrov and H. Sun, Opt. Lett., 2009, 34, 3340.

10 C. Schriever, C. Bohley and R. B. Wehrspohn, Opt. Lett., 2010, $35,273$.

11 S. A. Mitchell, M. Mehendale, D. M. Villeneuve and R. Boukherroub, Surf. Sci., 2001, 448, 367.

12 M. Cazzanelli, F. Bianco, E. Borga, G. Pucker, M. Ghulinyan, E. Degoli, E. Luppi, V. Veniard, S. Ossicini, D. Modotto, S. Wabnitz, R. Pierobon and L. Pavesi, Nat. Mater., 2012, 11, 148.

13 E. F. Pecora, A. Capretti, G. Miano and L. Dal Negro, Appl. Phys. Lett., 2013, 102, 141114.

14 M. E. Groenert, C. W. Leitz, A. J. Pitera, V. Yang, H. Lee, R. J. Ram and E. A. Fitzgerald, J. Appl. Phys., 2003, 93, 362.

15 T. Martensson, C. P. T. Svensson, B. A. Wacaser, M. W. Larsson, W. Seifert, K. Deppert, A. Gustafsson, L. R. Wallenberg and L. Samuelson, Nano Lett., 2004, 4, 1987.

16 V. Mourik, K. Zuo, S. M. Frolov, S. R. Plissard, E. P. A. M. Bakkers and L. P. Kouwenhoven, Science, 2013, 336, 1003. 
17 A. M. Morales and C. M. Lieber, Science, 1998, 279, 208.

18 M. S. Gudiksen, L. J. Lauhon, J. Wang, D. C. Smith and C. M. Lieber, Nature, 2002, 415, 617.

19 S. A. Dayeh, E. T. Yu and D. Wang, Nano Lett., 2007, 7, 2486.

20 A. Irrera, E. F. Pecora and F. Priolo, Nanotechnology, 2009, 20, 135601.

21 E. F. Pecora, N. Lawrence, P. Gregg, J. Trevino, P. Artoni, A. Irrera, F. Priolo and L. Dal Negro, Nanoscale, 2012, 4, 2863.

22 K. A. Dick, K. Deppert, M. W. Larsson, T. Martensson, W. Seifert, L. R. Wallenberg and L. Samuelson, Nat. Mater., 2004, 3, 380 .

23 X. Y. Kong, Y. Ding, R. Yang and Z. L. Wang, Science, 2004, 303, 1348.

24 H. Zhang, G. P. Cao, Z. Y. Wang, Y. S. Yang, Z. J. Shi and Z. N. Gu, Nano Lett., 2008, 8, 2664.

25 Y. B. Li, Y. Bando and D. Golberg, Appl. Phys. Lett., 2003, 82, 1962.

26 S. Conesa-Boj, E. Russo-Averchi, A. Dalmau-Mallorqui, J. Trevino, E. F. Pecora, C. Forestiere, A. Handin, M. Ek, L. Zweifel, L. R. Wallenberg, D. Ruffer, M. Heiss, D. Troadec, L. Dal Negro, P. Caroff and A. Fontcuberta i Morral, ACS Nano, 2012, 6, 10982.

27 H. Xiaoying, L. Jing, Z. Yong and A. Mascarenhas, J. Am. Chem. Soc., 2003, 125, 7049.

28 A. Irrera, P. Artoni, F. Iacona, E. F. Pecora, G. Franzò, M. Galli, B. Fazio and F. Priolo, Nanotechnology, 2012, 23, 075204.

29 V. R. Almeida, C. A. Barrios, R. R. Panepucci and M. Lipson, Nature, 2004, 431, 1081.

30 Q. Wan, K. Yu, T. H. Wang and C. L. Lin, Appl. Phys. Lett., 2003, 83, 2253.

31 C. Forestiere, G. Iadarola, G. Rubinacci, A. Tamburrino, L. Dal Negro and G. Miano, J. Opt. Soc. Am. A, 2012, 29, 2314.
32 R. Harrington, Time-Harmonic Electromagnetic Fields, Springer, 2007.

33 A. Poggio and E. Miller, Integral equation solutions for three dimensional scattering problem, in Computer Techniques for Electromagentics, ed. R. Mittra, Pergamon Press, NY, 1973, ch. 4.

34 E. Russo-Averchi, A. Dalmau-Mallorquí, I. Canales-Mundet,

G. Tütüncüoğlu, E. Alarcon-Llado, M. Heiss, D. Rüffer, S. Conesa-Boj, P. Caroff and A. Fontcuberta i Morral, Nanotech., 2013, in press.

35 S. Hertenberger, D. Rudolph, M. Bichler, J. J. Finley, G. Abstreiter and G. Koblmüller, J. App. Phys., 2010, 108, 114316.

36 M. T. Borgström, G. Immink, B. Ketelaars, R. Algra and E. P. A. M. Bakkers, Nat. Nanotechnol., 2007, 2, 541.

37 B. Bauer, A. Rudolph, M. Soda, A. Fontcuberta i Morral, J. Zweck, D. Schuh and E. Reiger, Nanotechnology, 2010, 21, 435601.

38 M. Reid, I. V. Cravetchi and R. Fedosejevs, Phys. Rev. B: Condens. Matter Mater. Phys., 2005, 72, 035201.

39 G. F. Walsh and L. Dal Negro, Nano Lett., 2013, 13, 3111.

40 R. S. Jacobsen, K. N. Andersen, P. I. Borel, J. Fage-Pedersen, L. H. Frandsen, O. Hansen, M. Kristensen, A. V. Lavrinenko, G. Moulin, H. Ou, C. Peucheret, B. Zsigri and A. Bjarklev, Nature, 2006, 441, 199.

41 M. Galli, D. Gerace, K. Welna, T. F. Krauss, L. O'Faolain, G. Guizzetti and L. C. Andreani, Opt. Express, 2010, 18, 26613.

42 B. Chmielak, M. Waldow, C. Matheisen, C. Ripperda, J. Bolten, T. Wahlbrink, M. Nagel, F. Merget and H. Kurz, Opt. Express, 2011, 19, 17212.

43 J. S. Levy, M. A. Foster, A. L. Gaeta and M. Lipson, Opt. Express, 2011, 19, 11415.

44 Y. R. Shen, Appl. Phys. B, 1999, 68, 295. 